DOI: $10.15393 / \mathrm{j} 3$.art.2021.9530

UDC 517.538.5

\author{
M. A. Komarov
}

\title{
RATIONAL APPROXIMATIONS OF LIPSCHITZ FUNCTIONS FROM THE HARDY CLASS ON THE LINE
}

\begin{abstract}
We study a rate of uniform approximations on the real line of summable Lipschitz functions $f$ having a summable Hilbert transform $\mathrm{Hf}$ by normalized logarithmic derivatives of rational functions. Inequalities between different metrics of the logarithmic derivatives of algebraic polynomials on the line are also considered.

Key words: logarithmic derivative of a rational function, simple partial fraction, Hilbert transform, uniform approximation, inequality between different metrics
\end{abstract}

2010 Mathematical Subject Classification: 41A20, 41A25

1. Main result. Denote the Hilbert transform of a function $f$ by $H f$,

$$
H f(x):=\frac{1}{\pi} \lim _{\varepsilon \rightarrow+0} \int_{|t-x|>\varepsilon} \frac{f(t)}{t-x} d t, \quad-\infty<x<+\infty .
$$

In this paper, by using the results of [10] and certain properties of the Hilbert transform, we obtain upper bounds for uniform approximations on $\mathbb{R}$ of a sufficiently wide subclass of real-valued functions $f$ by rational fractions $R_{n}, n=2,3, \ldots$, of the special form

$$
R_{n}(x)=\frac{C}{n}\left(\frac{P^{\prime}(x)}{P(x)}-\frac{Q^{\prime}(x)}{Q(x)}\right), \quad C=C(f)>0,
$$

where $P, Q$ are real polynomials of degree $n-1$. Here the expression in brackets is a difference of the so-called simple partial fractions

$$
\sum_{k=1}^{n-1} \frac{1}{x-z_{k}}
$$

(C) Petrozavodsk State University, 2021 
Setting $r_{n-1}=P / Q$, we represent the fraction (1) in the form of the normalized logarithmic derivative of a rational function of degree $n-1$ :

$$
R_{n}(x)=\frac{C}{n} \cdot \frac{r_{n-1}^{\prime}(x)}{r_{n-1}(x)}, \quad \operatorname{deg} R_{n}=2 n-2 .
$$

Further we denote $\|\cdot\|_{p}:=\|\cdot\|_{L^{p}(\mathbb{R})}(1 \leqslant p \leqslant \infty)$ and $L:=L^{1}(\mathbb{R})$. We write $f \in \operatorname{Lip}_{\alpha}\{A ; E\}$, if a function $f$ is defined on a set $E \subseteq \mathbb{R}$ and there are constants $\alpha \in(0,1]$ and $A>0$, such that

$$
\left|f\left(x_{1}\right)-f\left(x_{2}\right)\right| \leqslant A\left|x_{1}-x_{2}\right|^{\alpha}
$$

for any two points $x_{1}, x_{2} \in E$. By $C_{0}(\mathbb{R})$ we denote the class of continuous on $\mathbb{R}$ functions having the zero limit as $x \rightarrow \pm \infty$.

Theorem 1. Let $n=2,3, \ldots$ Let a real-valued function $f$ belong to $C_{0}(\mathbb{R})$ and let the following conditions hold:

1) $f \in \operatorname{Lip}_{\alpha}\{A ; \mathbb{R}\}$ with some $\alpha \in(0,1), A>0$,

2) $f \in L, H f \in L$.

Then there are real polynomials $Q_{1}, Q_{2}$ of degree $n-1$, such that

$$
\left|f(x)-\frac{\|H f\|_{1}}{4 \pi n} \frac{r^{\prime}(x)}{r(x)}\right|<\frac{c(f)}{n^{\alpha /(1+\alpha)}}, \quad-\infty<x<+\infty,
$$

where $r(x)=Q_{1}(x) / Q_{2}(x) ; c(f)>0$ is a constant depending only on $f$.

Theorem 1 is proved in Section 3; we show that the constant

$$
c(f)=4 A(\alpha \pi)^{-1} \mathrm{~B}\left(\frac{\alpha}{2}, \frac{1-\alpha}{2}\right)+2 \alpha^{-1}\|H f\|_{1}
$$

is suitable (here $\mathrm{B}(x, y)$ is Euler's beta-function). Our proof uses the wellknown implication: $f \in \operatorname{Lip}_{\alpha}\{A ; \mathbb{R}\}(\alpha \in(0,1)) \Rightarrow H f \in \operatorname{Lip}_{\alpha}\{\tilde{A} ; \mathbb{R}\}$. Here the restriction $\alpha<1$ is essential; nevertheless, if the assumptions of Theorem 1 hold for $\alpha=1$ and, additionally, $H f \in \operatorname{Lip}_{1}\{\tilde{A} ; \mathbb{R}\}$, then the estimate given in Theorem 1 holds with the bound

$$
c(f) / \sqrt{n}, \quad c(f):=4 \tilde{A}+2\|H f\|_{1} .
$$

For a small $\alpha$, the order of approximation $O\left(n^{-\alpha /(1+\alpha)}\right) \approx O\left(n^{-\alpha}\right)$, established by the theorem, cannot be essentially improved in the following sense: if approximations of a function $f$ by the class of all rational functions of degree $2 n-2$ have order $O\left(n^{-\alpha_{0}-\varepsilon}\right.$ ) with $\alpha_{0} \in(\alpha, 1)$ (while $\varepsilon>0$ is 
arbitrarily small) for all $n=2,3, \ldots$, then, by the Gonchar converse theorem [6], $f$ satisfies the Lipschitz condition of degree $\alpha_{0}$ almost everywhere on $\mathbb{R}$ (in contrast to the condition $f \in \operatorname{Lip}_{\alpha}\{A ; \mathbb{R}\}$, where $\alpha<\alpha_{0}$ ).

Borodin and Kosukhin [2] have proved that any function $f \in C_{0}(\mathbb{R})$ can be approximated uniformly on $\mathbb{R}$ by sums of the form (2) with poles $z_{k}$ outside any given strip $|\operatorname{Im} z|<$ const. In our construction, all poles of the approximating functions $R_{n}$ (i.e., the zeros of the polynomials $Q_{1}$, $\left.Q_{2}\right)$ lie on the two lines $\operatorname{Im} z= \pm n^{-1 /(1+\alpha)}$, so that $\left|\operatorname{Im} z_{k}\right| \rightarrow 0$ as $n \rightarrow \infty$.

Some estimates of uniform approximations on $\mathbb{R}$ of certain functions $f$ by differences of simple partial fractions were obtained in [9]. For example, an order of such approximations is $O\left(n^{-1}\right)$ if a function $f$ has the form

$$
f(x)=\frac{x}{\left(1+x^{2}\right)^{2}} F\left(\frac{1-x^{2}}{1+x^{2}}\right), \quad-\infty<x<+\infty,
$$

with some function $F(t) \in \operatorname{Lip}_{1}\{A ;[-1,1]\}$. Concerning the uniform approximation rate by simple partial fractions themselves (not by their differences) on the whole real axis recall the result by Danchenko [4]: for any function $f$ of the form

$$
f(x)=f_{a}(x)=-\frac{1}{x-a}, \quad a \in \mathbb{C} \backslash \mathbb{R},
$$

and sufficiently large $n \geqslant n_{0}(a)$ there is a complex polynomial $P$ of degree $n$, such that

$$
\left|f_{a}(x)-P^{\prime}(x) / P(x)\right|<C \cdot \ln \ln n / \ln n, \quad-\infty<x<+\infty,
$$

where $C>0$ is a constant depending only on $a$ (the order of approximation cannot be improved). At the end of Section 3, we discuss the rate of the uniform approximation by normalized simple partial fractions.

2. Some remarks on the assumptions of Theorem 1. The class of functions $f$, such that $f \in L$ and $H f \in L$, is called [12, p. 165] the Hardy class $H_{1}(-\infty, \infty)$. Thus, the second condition of Theorem 1 can be written as follows: $f \in H_{1}(-\infty, \infty)$. For example, the class $H_{1}(-\infty, \infty)$ contains the derivative $R^{\prime}$ of any bounded on $\mathbb{R}$ rational function $R$, because of the Rusak inequality

$$
\left\|R^{\prime}\right\|_{1}+\left\|H\left(R^{\prime}\right)\right\|_{1} \leqslant 4 \pi n\|R\|_{\infty}, \quad n=\operatorname{deg} R
$$

(see [12, p. 165]). Further nontrivial examples of functions $f \in H_{1}(-\infty, \infty)$ can be found in the paper by Kober [8]. 
Protasov [13] described the class $V_{p}=V_{p}(\mathbb{R})$ of functions $f \in L^{p}(\mathbb{R})$, $p \in(1, \infty)$, that can be approximated in $L^{p}(\mathbb{R})$ by sums of the form

$$
\sum_{k=1}^{N} \frac{p_{k}}{x-z_{k}}, \quad p_{k} \geqslant 0 .
$$

In particular, [13, Corollary 1], if a function $f$ belongs to $L^{p}(\mathbb{R})$ and is real-valued, then $f \in V_{p}$ if and only if $H f(x) \geqslant 0$ for almost all $x \in \mathbb{R}$.

Let us show that a nonzero function $f$, satisfying the conditions of Theorem 1, cannot be approximated by sums $(3)$ in $L^{p}(\mathbb{R})$.

Proposition 1. Let a real-valued function $f$ belong to $L^{p}(\mathbb{R}), p \in(1, \infty)$. Then $f \in V_{p} \cap H_{1}(-\infty, \infty)$ if and only if $f(x)=0$ a.e.

Proof. The sufficient condition is obvious. To prove the necessary condition, we first recall the result of Kober [8, Theorem 1]:

$$
f \in L, \quad H f \in L \Rightarrow \int_{-\infty}^{\infty} f(x) d x=0 .
$$

On the other hand (Hille and Tamarkin, see [8, Lemma 2]), we have ${ }^{1}$

$$
f \in L, \quad H f \in L \Rightarrow H H f=-f \text { a.e. }
$$

Thus, if $f \in H_{1}(-\infty, \infty)$, then $\tilde{f}:=H f \in L$ and, by (5), $H \tilde{f}=-f \in L$; by applying (4) to the function $\tilde{f}$, we get

$$
f \in H_{1}(-\infty, \infty) \Rightarrow \int_{-\infty}^{\infty} H f(x) d x=0 .
$$

But if $f \in V_{p}$, then $\operatorname{Hf}(x) \geqslant 0$ a.e. [13]. Hence, for any function $f \in V_{p} \cap H_{1}(-\infty, \infty)$ we have $H f(x)=0$ a.e. Therefore, $f(x)=0$ a.e. by $f=-H H f$, see (5).

Let us formulate another simple observation concerning the class $V_{p}$. Proposition 2. Let an even real-valued function $f$ belong to $L^{p}(\mathbb{R})$, $p \in(1, \infty)$. Then $f \in V_{p}$ if and only if $f(x)=0$ a.e.

\footnotetext{
${ }^{1}$ Of course, we also have $H H f=-f$ (a.e.) due to $f \in L^{p}(\mathbb{R}), p>1[7$, p. 148].
} 
Proof. Indeed, the Hilbert transform $H f$ of an even function $f$ is odd (see [7, p. 146]). But if an odd function $H f(x)$ is non-negative (a.e.), then $H f(x)=0$ (a.e.). Finally, we use, again, the formula $f=-H H f$, which is correct due to $f \in L^{p}(\mathbb{R})$.

The results of Danchenko [4] yield that the functions $f_{a}(x)$ (see Section 1) cannot be approximated by simple partial fractions in $L^{p}(\mathbb{R})$ with finite $p$. In particular, this remark is also true for the real-valued function

$$
g(x):=-\frac{2 x}{x^{2}+1} \equiv-\frac{1}{x+i}-\frac{1}{x-i} .
$$

At the end of Section 4, we establish that the normalized logarithmic derivatives $Q^{\prime}(x) /(n Q(x))$ of real polynomials $Q(x)$ rapidly converge to $g(x)$ on the line in $L^{p}(\mathbb{R})$ with any $1<p \leqslant \infty$. Note that $g \in V_{p}$ for all $1<p<\infty$ by the theorem of Protasov, because

$$
H g(x)=\frac{i}{x+i}+\frac{-i}{x-i}=\frac{2}{x^{2}+1} \geqslant 0
$$

(see [7, p. 104] for explicit values of $H\left((x+i \alpha)^{-1}\right)$ with nonzero $\left.\alpha\right)$.

Although the class $V_{p}$ is narrow, Protasov has showed [13, Remark 1] that any function $f \in L^{p}(\mathbb{R}), p \in(1, \infty)$, can be approximated in $L^{p}(\mathbb{R})$ by differences of sums of the form (3). Obviously, the normalized logarithmic derivatives of rational functions, see (1), belong to the space of such differences.

3. Proof of Theorem 1. Put $\tilde{f}=H f$. Since $f \in \operatorname{Lip}_{\alpha}\{A ; \mathbb{R}\}$ with $\alpha \in(0,1)$, it follows by the theorem of Aleksandrov [1] that

$$
\tilde{f} \in \operatorname{Lip}_{\alpha}\{\tilde{A} ; \mathbb{R}\}, \quad \tilde{A}=A \pi^{-1} \mathrm{~B}\left(\frac{\alpha}{2}, \frac{1-\alpha}{2}\right) .
$$

Let us write the real-valued function $\tilde{f}$ in the form $\tilde{f}=\tilde{f}_{1}-\tilde{f}_{2}$,

$$
\tilde{f}_{1}(x):=\max \{\tilde{f}(x) ; 0\} \geqslant 0, \quad \tilde{f}_{2}(x):=\max \{-\tilde{f}(x) ; 0\} \geqslant 0 .
$$

Both functions $\tilde{f}_{k}$ also belong to the class $\operatorname{Lip}_{\alpha}\{\tilde{A} ; \mathbb{R}\}$ : for example, the identity $\tilde{f}_{1}(x)=(\tilde{f}(x)+|\tilde{f}(x)|) / 2$ and the triangle inequality yield $\left|\tilde{f}_{1}\left(x_{1}\right)-\tilde{f}_{1}\left(x_{2}\right)\right| \leqslant \frac{1}{2}\left|\tilde{f}\left(x_{1}\right)-\tilde{f}\left(x_{2}\right)\right|+\frac{1}{2}|| \tilde{f}\left(x_{1}\right)|-| \tilde{f}\left(x_{2}\right)|| \leqslant\left|\tilde{f}\left(x_{1}\right)-\tilde{f}\left(x_{2}\right)\right|$.

By the assumptions of the theorem, $\tilde{f} \in L$. Hence, $\tilde{f}_{1}, \tilde{f}_{2} \in L$ and

$$
\|\tilde{f}\|_{1}=\int_{-\infty}^{\infty} \tilde{f}_{1}(x) d x+\int_{-\infty}^{\infty} \tilde{f}_{2}(x) d x=\left\|\tilde{f}_{1}\right\|_{1}+\left\|\tilde{f}_{2}\right\|_{1} .
$$


From this, we get $\left\|\tilde{f}_{1}\right\|_{1}=\left\|\tilde{f}_{2}\right\|_{1}=\frac{1}{2}\|\tilde{f}\|_{1}$ using the formula (6).

Further, we can assume $\|\tilde{f}\|_{1}>0$. Both functions

$$
F_{k}(x):=\tilde{f}_{k}(x) /\left\|\tilde{f}_{k}\right\|_{1}=2 \tilde{f}_{k}(x) /\|\tilde{f}\|_{1}, \quad k=1 ; 2,
$$

are non-negative and

$$
\left\|F_{k}\right\|_{1}=1, \quad F_{k} \in \operatorname{Lip}_{\alpha}\left\{A^{*} ; \mathbb{R}\right\} \quad\left(A^{*}:=2 \tilde{A} /\|\tilde{f}\|_{1}\right) .
$$

By [10, Theorem 3], there are real polynomials $Q_{1}, Q_{2}$ of degree $n-1$, such that

$$
\left|H F_{k}(x)+\frac{1}{2 \pi n} \frac{Q_{k}^{\prime}(x)}{Q_{k}(x)}\right|<\frac{2 A^{*}+2}{\alpha n^{\alpha /(1+\alpha)}}, \quad-\infty<x<\infty, \quad k=1 ; 2 .
$$

Namely (see [10, Lemma 2]), we can take

$$
Q_{k}(x)=\prod_{j=1}^{n-1}\left(\left(x_{k, j}-x\right)^{2}+n^{-2 /(1+\alpha)}\right), \quad k=1 ; 2,
$$

where the points $x_{k, 0}=-\infty<x_{k, 1}<\ldots<x_{k, n-1}<x_{k, n}=\infty$ are defined by

$$
\int_{x_{k, j}}^{x_{k, j+1}} F_{k}(x) d x=\frac{1}{n}, \quad j=0, \ldots, n-1 .
$$

Hence,

$$
\left|H F_{1}(x)-H F_{2}(x)+\frac{1}{2 \pi n} \frac{r^{\prime}(x)}{r(x)}\right|<\frac{4 A^{*}+4}{\alpha n^{\alpha /(1+\alpha)}}, \quad-\infty<x<+\infty,
$$

where $r(x):=Q_{1}(x) / Q_{2}(x)$ and

$$
H F_{1}(x)-H F_{2}(x)=2\|\tilde{f}\|_{1}^{-1} H\left(\tilde{f}_{1}(x)-\tilde{f}_{2}(x)\right)=-2\|H f\|_{1}^{-1} f(x)
$$

by (5). Theorem 1 is proved.

By using very similar arguments, we easily obtain the following assertion, which complements the theorem in the case when $f \notin L$ and $f \in L^{p}(\mathbb{R}), p>1$.

Proposition 3. Let $p \in(1, \infty), n=2,3, \ldots$ Let a real-valued function $f$ belong to $C_{0}(\mathbb{R}) \cap L^{p}(\mathbb{R})$. If the function $H f$ is nonnegative, $H f \in L$ 
and $\operatorname{Hf} \in \operatorname{Lip}_{\alpha}\{\tilde{A} ; \mathbb{R}\}$ with some $\alpha \in(0,1], \tilde{A}>0$, then there is a real polynomial $Q$ of degree $n-1$, such that

$$
\left|f(x)-\frac{\|H f\|_{1}}{2 \pi n} \frac{Q^{\prime}(x)}{Q(x)}\right|<2 \cdot \frac{\tilde{A}+\|H f\|_{1}}{\alpha n^{\alpha /(1+\alpha)}}, \quad-\infty<x<+\infty .
$$

Note that any function $f$, satisfying the conditions of Proposition 3, belongs to $V_{p}$. If, moreover, $f \not \equiv 0$, then $f \notin L$ (sf. Proposition 1$)$.

Proof. Assume that $d:=\|H f\|_{1}>0$ and set $F(x)=H f(x) / d$. The function $F$ is nonnegative and $\|F\|_{1}=1, F \in \operatorname{Lip}_{\alpha}\{\tilde{A} / d ; \mathbb{R}\}$. By [10, Theorem 3], there is a real polynomial $Q$ of degree $n-1$, such that

$$
\left|H F(x)+\frac{1}{2 \pi n} \frac{Q^{\prime}(x)}{Q(x)}\right|<\frac{2 \tilde{A}+2 d}{\alpha n^{\alpha /(1+\alpha)} d}, \quad-\infty<x<+\infty .
$$

By $f \in L^{p}(\mathbb{R})$, we have $f=-H H f$. Hence, $H F(x) \equiv-f(x) / d$, and the assertion follows. The case $d=0$ is trivial $(f \equiv 0)$.

4. Inequalities between different metrics for simple partial fractions. Nikol'skii inequalities for simple partial fractions

$$
\rho_{n}(z)=\sum_{k=1}^{n} \frac{1}{z-z_{k}}
$$

were studied by many authors (see, for example, [5], [3] and references therein). Let us recall one result of the paper by Chunaev and Danchenko [3], stated as Theorem 4.5: for any $z_{1}, \ldots, z_{n} \in \mathbb{C} \backslash \mathbb{R}$ and $1<p<q \leqslant \infty$,

$$
\left\|\rho_{n}\right\|_{q}^{q^{\prime}} \leqslant 2^{q^{\prime}-p^{\prime}}\left(\frac{\kappa_{p}}{\pi}\right)^{p^{\prime} q^{\prime}\left(\frac{1}{p}-\frac{1}{q}\right)}\left(1+c_{p}\right)^{p^{\prime}}\left\|\rho_{n}\right\|_{p}^{p^{\prime}}
$$

where $\kappa_{p}$ is a unique natural number, which belongs to $\left[\frac{p}{2}, \frac{p}{2}+1\right)$,

$$
\frac{1}{q}+\frac{1}{q^{\prime}}=1=\frac{1}{p}+\frac{1}{p^{\prime}}, \quad c_{p}= \begin{cases}\tan \frac{\pi}{2 p}, & 1<p \leqslant 2, \\ \cot \frac{\pi}{2 p}, & 2 \leqslant p<\infty,\end{cases}
$$

$c_{p} \geqslant 1$ is the norm of the Hilbert transform in $L^{p}(\mathbb{R})$. A similar inequality with a bigger constant was first obtained by Danchenko and Dodonov in the paper [5], where the authors raised the problem of finding a better upper bound for the ratio $\left\|\rho_{n}\right\|_{q}^{q^{\prime}} /\left\|\rho_{n}\right\|_{p}^{p^{\prime}}$. 
Thus, our goal is to improve the constant factor in the estimate (7).

If all $z_{k}$ are non-real, then $\rho_{n}(x)$ is bounded on the real line:

$$
M:=\left\|\rho_{n}\right\|_{\infty}<\infty
$$

Putting $q=\infty$ in (7), we get

$$
M \leqslant 2^{1-p^{\prime}}\left(\frac{\kappa_{p}}{\pi}\right)^{p^{\prime} / p}\left(1+c_{p}\right)^{p^{\prime}}\left\|\rho_{n}\right\|_{p}^{p^{\prime}}, \quad 1<p<\infty .
$$

But

$$
1-p^{\prime}=p^{\prime}\left(\frac{1}{p^{\prime}}-1\right)=-\frac{p^{\prime}}{p}
$$

therefore, (8) can be written in the form

$$
M \leqslant\left(\frac{\kappa_{p}}{2 \pi}\right)^{p^{\prime} / p}\left(1+c_{p}\right)^{p^{\prime}}\left\|\rho_{n}\right\|_{p}^{p^{\prime}}, \quad 1<p<\infty .
$$

Now, let $q<\infty$. Since $\left|\rho_{n}(x)\right| \leqslant M$ at points $x \in \mathbb{R}$, we see that

$$
\int_{-a}^{a}\left|\rho_{n}(x)\right|^{q} d x=\int_{-a}^{a}\left|\rho_{n}(x)\right|^{q-p}\left|\rho_{n}(x)\right|^{p} d x \leqslant M^{q-p} \int_{-a}^{a}\left|\rho_{n}(x)\right|^{p} d x
$$

for $q>p$ and any $a>0$. Letting $a \rightarrow \infty$, we get

$$
\left\|\rho_{n}\right\|_{q}^{q} \leqslant M^{q-p}\left\|\rho_{n}\right\|_{p}^{p}, \quad 1<p<q,
$$

because $\rho_{n}$ belongs to all the spaces $L^{p}(\mathbb{R}), p>1$.

Using the estimate (9) and the transformation

$$
(q-p) \frac{p^{\prime} q^{\prime}}{q}=p q\left(\frac{1}{p}-\frac{1}{q}\right) \frac{p^{\prime} q^{\prime}}{q}=p\left(p^{\prime}-q^{\prime}\right)
$$

we obtain

$$
\left\|\rho_{n}\right\|_{q}^{q^{\prime}} \leqslant M^{(q-p) q^{\prime} / q}\left\|\rho_{n}\right\|_{p}^{p q^{\prime} / q} \leqslant\left(\frac{\kappa_{p}}{2 \pi}\left(1+c_{p}\right)^{p}\right)^{p^{\prime}-q^{\prime}}\left\|\rho_{n}\right\|_{p}^{p\left(p^{\prime}-q^{\prime}\right)+p q^{\prime} / q} .
$$

Observe that

$$
p\left(p^{\prime}-q^{\prime}\right)+\frac{p q^{\prime}}{q}=p q^{\prime}\left(\frac{p^{\prime}}{q^{\prime}}-1+\frac{1}{q}\right)=p p^{\prime}\left(1-\frac{1}{p^{\prime}}\right)=p^{\prime} .
$$


Thus, we have proved the following result:

Theorem 2. For any simple partial fraction $\rho_{n}$ without poles on $\mathbb{R}$ :

$$
\left\|\rho_{n}\right\|_{q}^{q^{\prime}} \leqslant\left(\frac{\kappa_{p}}{2 \pi}\left(1+c_{p}\right)^{p}\right)^{p^{\prime}-q^{\prime}}\left\|\rho_{n}\right\|_{p}^{p^{\prime}}, \quad 1<p<q \leqslant \infty .
$$

For $q=\infty$, the estimate (10) coincides with the result of Chunaev and Danchenko (7), because of the equality $p\left(p^{\prime}-1\right)=p^{\prime}$. However, for any $q<\infty$, Theorem 2 is stronger than (7), since in this case

$$
p\left(p^{\prime}-q^{\prime}\right)=p^{\prime}\left(p^{\prime}-q^{\prime}\right) /\left(p^{\prime}-1\right)<p^{\prime}
$$

and, therefore,

$$
\left(1+c_{p}\right)^{p\left(p^{\prime}-q^{\prime}\right)}<\left(1+c_{p}\right)^{p^{\prime}}
$$

Even more, in contrast to (7), the estimate (10) has the following important property: the left-hand side of the estimate tends to the right-hand side as $q \rightarrow p$.

Our next purpose is to establish some $(q, \infty)$ Nikol'skii inequalities for differences of simple partial fractions. Let $\Theta$ be a weak norm of the Hilbert transform, i.e., the smallest possible value of a constant $C$ in the Kolmogorov inequality

$$
m(\{x \in \mathbb{R}:|H f(x)| \geqslant \delta\}) \leqslant C\|f\|_{1} / \delta
$$

where $f$ is any real-valued summable function and $m(E)$ denotes the Lebesgue measure of a set $E \subset \mathbb{R}$. Recall that [7, p. 338]

$$
\Theta=\frac{\pi^{2} / 8}{1-3^{-2}+5^{-2}-\ldots}=1.347 \ldots
$$

It was proved in [10] that if $r$ is a real rational function of degree $n$ and $\mu(r, \delta):=m\left(\left\{x \in \mathbb{R}:\left|r^{\prime}(x) / r(x)\right| \geqslant \delta\right\}\right)$, then, for any $\delta>0$,

$$
\mu(r, \delta) \leqslant 2 \pi \Theta \cdot n / \delta
$$

where the constant $2 \pi \Theta$ cannot be replaced by a smaller value. Note that (11) can be formulated as follows: for any real rational function $r$ of degree $n$ and $\delta>0$ there is a set $E=E(r, \delta) \subset \mathbb{R}$, such that $m(E) \leqslant \delta$ and

$$
\left|r^{\prime}(x)\right| \leqslant 2 \pi \Theta \cdot \frac{n}{\delta}|r(x)|, \quad x \in \mathbb{R} \backslash E .
$$


The last estimate was first obtained (with a bigger factor $C \ln n$ instead of $2 \pi \Theta$ ) by Gonchar [6] and used by him in the proof of the converse theorem, mentioned in Section 1 above.

Estimates of the quantity $\mu(r, \delta)$ are well-known in the case of complex polynomials $r=P$ by the works of Macintyre and Fuchs, Govorov and Grushevskii and others (see details and references in [10]). For example, the famous result by Macintyre and Fuchs (1940) is

$$
\mu(P, \delta) \leqslant 2 e \cdot n / \delta, \quad n=\operatorname{deg} P \quad(\delta>0) .
$$

The best possible result [11] for real polynomials $P$ of degree $n$ is

$$
\mu(P, \delta) \leqslant \pi \cdot n / \delta \quad(\delta>0) .
$$

Using (11) and (12), we easily establish the following extension of theorem 3 of the paper [5], where the case of complex polynomials $r=P$ is considered:

Theorem 3. Let $1<q \leqslant \infty$ and let $E$ be an arbitrary bounded or unbounded segment of $\mathbb{R}$. Then, for any real rational function $r$ of degree $n$ without poles and zeros on $E$ we have

$$
\|R\|_{L^{q}(E)}^{q^{\prime}} \leqslant\left(2 \pi \Theta \cdot n q^{\prime}\right)^{q^{\prime} / q}\|R\|_{L^{\infty}(E)}, \quad 1 / q+1 / q^{\prime}=1,
$$

where $R(x)=r^{\prime}(x) / r(x)$. Moreover, if $r(x)=P(x)$ is a real polynomial of degree $n$, i.e., $R(x) \equiv \rho_{n}(x)$ is a real-valued simple partial fraction, then the constant $2 \pi \Theta$ in this estimate can be replaced by $\pi$.

Proof. Set $M=\|R\|_{L^{\infty}(E)}$. By the assumptions of the theorem, we have $M<\infty$ and $R \in L^{q}(E)$ for all $q>1$. Next, we have [7, p. 233]

$$
\|R\|_{L^{q}(E)}^{q}=q \int_{0}^{M} \tilde{\mu}(\delta) \delta^{q-1} d \delta, \quad \tilde{\mu}(\delta):=m(\{x \in E:|R(x)| \geqslant \delta\}) .
$$

But $\tilde{\mu}(\delta) \leqslant \mu(r, \delta)$, hence, by $(11)$ :

$$
\|R\|_{L^{q}(E)}^{q} \leqslant q \int_{0}^{M} 2 \pi \Theta \cdot n \cdot \delta^{q-2} d \delta=2 \pi \Theta \cdot n q^{\prime} M^{q-1},
$$

and the first assertion of the theorem follows. Analogously, the second assertion follows from (12). 
Corollary. Let $n=2,3, \ldots$ and $g(x)=-2 x /\left(x^{2}+1\right)$. There is a real polynomial $Q$ of degree $n-1$, such that for every $1<q \leqslant \infty$

$$
\left\|g-\frac{1}{n} \frac{Q^{\prime}}{Q}\right\|_{q}^{q^{\prime}}<\frac{c_{q}}{\sqrt{n}}, \quad c_{q}:=4(\pi+1)\left(3 \pi q^{\prime}\right)^{q^{\prime} / q}, \quad 1 / q+1 / q^{\prime}=1 .
$$

Proof. Recall that $H g(x)=2 /\left(x^{2}+1\right)$ (see Section 2), therefore,

$$
H g \in L, \quad\|H g\|_{1}=2 \pi, \quad H g \in \operatorname{Lip}_{1}\{2 ; \mathbb{R}\} .
$$

By applying Proposition 3, we get existence of a real polynomial $Q$ of degree $n-1$, such that

$$
\|\Delta\|_{\infty}<\frac{4(\pi+1)}{\sqrt{n}} \equiv \frac{c_{\infty}}{\sqrt{n}} \quad\left(\Delta(x):=\frac{1}{n} \frac{Q^{\prime}(x)}{Q(x)}-g(x)\right) .
$$

Now consider the logarithmic derivative $R(x):=h^{\prime}(x) / h(x)$, where

$$
h(x):=Q(x)\left(x^{2}+1\right)^{n}
$$

is a real polynomial of degree $(n-1)+2 n<3 n$. But $R(x) \equiv n \Delta(x)$; hence, by Theorem 3, we have

$$
\begin{gathered}
\|\Delta\|_{q}^{q^{\prime}}=n^{-q^{\prime}}\|R\|_{q}^{q^{\prime}} \leqslant n^{-q^{\prime}}\left(\pi \cdot \operatorname{deg} h \cdot q^{\prime}\right)^{q^{\prime} / q}\|R\|_{\infty} \leqslant \\
\leqslant n^{1-q^{\prime}}\left(\pi \cdot 3 n \cdot q^{\prime}\right)^{q^{\prime} / q}\|\Delta\|_{\infty}=n^{1-q^{\prime}+q^{\prime} / q}\left(3 \pi q^{\prime}\right)^{q^{\prime} / q}\|\Delta\|_{\infty},
\end{gathered}
$$

where $1-q^{\prime}+q^{\prime} / q=0$. Thus, the result follows from this and the previous estimate of $\|\Delta\|_{\infty}$.

Acknowledgment. The author is grateful to the referees for their helpful comments and suggestions.

\section{References}

[1] Aleksandrov A. B., Norm of the Hilbert transformation in a space of Hölder functions. Funct. Anal. Appl., 1975, vol. 9, no. 2, pp. 94-96.

DOI: https://doi.org/10.1007/BF01075444

[2] Borodin P. A., Kosukhin O. N., Approximation by the simplest fractions on the real axis. Moscow Univ. Math. Bull., 2005, vol. 60, no. 1, pp. 1-6. https://zbmath.org/?q=an\%3A1101.41016 
[3] Chunaev P. V., Danchenko V. I., Quadrature formulas with variable nodes and Jackson-Nikolskii inequalities for rational functions. J. Approx. Theory, 2018, vol. 228, pp. 1-20.

DOI: https://doi.org/10.1016/j.jat.2017.12.004

[4] Danchenko V. I., Estimates of the distances from the poles of logarithmic derivatives of polynomials to lines and circles. Sb. Math., 1995, vol. 82, no. 2 , pp. $425-440$.

DOI: https://doi.org/10.1070/SM1995v082n02ABEH003573

[5] Danchenko V. I., Dodonov A. E., Estimates for $L_{p}$-norms of simple partial fractions. Russian Math. (Iz. VUZ), 2014, vol. 58, no. 6, pp. 6-15.

DOI: https://doi.org/10.3103/S1066369X14060024

[6] Gonchar A. A., Converse theorems concerning best approximations by rational functions. Izv. Akad. Nauk SSSR Ser. Mat., 1961, vol. 25, pp. 347-356 (in Russian).

DOI: https://mathscinet.ams .org/mathscinet-getitem?mr=125382

[7] King F. W., Hilbert transforms. Vol. 1. Encyclopedia of mathematics and its applications (vol. 124). Cambridge Univ. Press, Cambridge, 2009.

DOI: https://doi.org/10.1017/CB09780511721458

[8] Kober H., A note on Hilbert's operator. Bull. Amer. Math. Soc., 1942, vol. 48 , pp. $421-427$.

DOI: https://doi.org/10.1090/S0002-9904-1942-07688-9

[9] Komarov M. A., Approximation by linear fractional transformations of simple partial fractions and their differences. Russian Math. (Iz. VUZ), 2018, vol. 62 , no. 3 , pp. $23-33$.

DOI: https://doi.org/10.3103/S1066369X18030040

[10] Komarov M. A., Distribution of the logarithmic derivative of a rational function on the line. Acta Math. Hungar., 2021, vol. 163, no. 2, pp. 623-639. DOI: https://doi.org/10.1007/s10474-020-01102-w

[11] Komarov M. A., Metric property of a real polynomial. Bull. London Math. Soc., 2021, vol. 53 , no. 2 , pp. $336-346$.

DOI: https://doi.org/10.1112/blms. 12421

[12] Petrushev P. P., Popov V. A., Rational approximation of real functions. Encyclopedia of mathematics and its applications (vol. 28). Cambridge Univ. Press, Cambridge, 1987.

DOI: https://doi.org/10.1017/CB09781107340756

[13] Protasov V. Yu. Approximation by simple partial fractions and the Hilbert transform. Izv. Math., 2009, vol. 73, no. 2, pp. 333-349.

DOI: https://doi.org/10.1070/IM2009v073n02ABEH002449 
Received December 15, 2020.

In revised form, April 09, 2021.

Accepted April 14, 2021.

Published online April 19, 2021.

Vladimir State University

Gor'kogo street 87, Vladimir 600000, Russia

E-mail: kami9@yandex.ru 